\title{
EL RIESGO DE CRUZAR LOS DARDANELOS
}

\author{
RICARDO H. ELÍA \\ Centro Islámico de la República Argentina
}

Resumen: Desde las anárquicas hordas persas de Jerjes a los flemáticos batallones británicos de Churchill, la empresa de cruzar los Dardanelos ha resultado no sólo ardua sino costosa y mortífera. Paradójicamente, el único indemne en saltar de Europa al Asia fue Alejandro el Grande al iniciar la victoriosa conquista del Imperio Aqueménida.

Palabras claves: Estrecho - paso - encrucijada - helespóntico - estratégico.

\section{THE RISK OF CROSSING THE DARDANELLES}

\begin{abstract}
From the anarchic Persian hordes of Xerxes to the phlegmatic British battalions of Churchill, the attempt to crossing the Dardanelles has resulted not only challenging but costly and deadly. Paradoxically, the only harmless to jump from Europe to Asia was Alexander the Great to begin the victorious conquest of the Achaemenid Empire.
\end{abstract}

Key words: Strait - underpass - crossroad - Hellespontic - strategic.

Recibido: 10.04.15 - Aceptado: 20.05.15

\section{Correspondencia: Ricardo H. Elía.}

Email: ricardohselia@yahoo.com.ar

Secretario de Cultura y Director del Departamento de Estudios Históricos del Centro Islámico de la República Argentina. Dirección postal: Av. San Juan 3053 (1233) Buenos Aires, Argentina. Tel. (54-11) 4931-3577 (interno 113). 
—1 estrecho de los Dardanelos comunica el mar Egeo con el mar interior 1000 y 6200 metros de anchura y tiene una profundidad media de unos 55 metros y una máxima de 103. El estrecho separa la península de Gallipoli (en la Turquía europea) de la Turquía asiática, y, con el Bósforo, es de suma importancia estratégica, ya que se trata de la única ruta naval del Mediterráneo al mar Negro, y viceversa.

\section{Desde Hero y Leandro a Lord Byron}

A lo largo de la historia los Dardanelos ${ }^{1}$, el Helesponto ${ }^{2}$ de los antiguos helenos, ha estado signado por la tragedia. Basta con remontarse al mito griego de Hero y Leandro para saber de qué estamos hablando. Se trata de un amor verdadero, profundo y apasionado que trasgredió las barreras sociales, culturales y geográficas y que emociona hasta el llanto. Hero era una sacerdotisa de Afrodita que vivía en una torre en Sestos $^{3}$, en la orilla europea del Helesponto.

1 Dardanelos deriva de Dárdanos En la mitología griega es el nombre de la ciudad fundada en el monte Ida (en la orilla asiática del estrecho) por Dárdano (el hijo de Zeus y Electra), de quien tanto la región como el pueblo tomaron su nombre. De Tros, el nieto de Dárdano, la gente consiguió el nombre adicional de troyanos y la región el nombre de Tróade. Ilo, el hijo de Tros, fundó una ciudad llamada Ilión, ciudad más comúnmente llamada Troya. El reino fue dividido entre Ilión y Dárdanos. Las ruinas de Dárdanos hoy se pueden localizar cerca de la ciudad turca de Çanakkale, en el camino hacia Troya.

2 Literalmente en griego antiguo, "Mar de Hele". En la mitología griega, Hele es hija del rey beocio Atamante y de su primera esposa Néfele. Vuelto a casar su padre, Ino (una de las hijas de Cadmo y Harmonía), su madrastra, urde por celos una trama contra ella y su hermano, Frixo, de forma que ambos van a ser sacrificados. Sin embargo, un carnero enviado por Néfele con el vellocino de oro se los llevó a ambos volando por los aires, hacia la Cólquide (hoy Georgia), donde solo llegó Frixo, pues Hele pereció al caer durante el vuelo al mar en el estrecho que separa al Mediterráneo del Mar Negro. En recuerdo de la joven desaparecida, el mar donde se ahogó llevó el nombre de Helesponto.

3 Sestos (en griego antiguo, Sestós) fue la principal ciudad del Quersoneso Tracio, situada en la parte más estrecha del Helesponto, en la orilla europea del estrecho de los Dardanelos, enfrente y al norte de Abidos — situada ésta al otro lado del Helesponto, en la costa asiática. El lugar actual de sus ruinas es Zemenic, cercana a la bahía turca de Ak Bashi Liman. 
Leandro, un joven de $\mathrm{Abidos}^{4}$ sobre la orilla anatólica del estrecho, se enamoró de ella. Los padres de ambos amantes se opusieron a que se casaran como era su deseo, y un día, hartos de sus encuentros a pesar de las indicaciones que les hacían, les prohibieron terminantemente cualquier contacto. Los enamorados idearon un plan para verse en secreto. Cada noche, Hero encendía un faro desde la torre, y ésta servía de guía para que Leandro, en la orilla opuesta, cruzase a nado y llegara hasta ella. Una noche, sin embargo, hubo un fuerte vendaval que apagó la luz encendida por Hero, por lo que Leandro perdió el camino y pereció ahogado. Las horas pasaron, y cuando Hero atisbó en la costa el cadáver de Leandro se arrojó desde la torre, suicidándose.

Esta epopeya fue evocada en un magnífico poema de 342 hexámetros por Museo el Gramático, un poeta griego que floreció entre los siglo V y VI d. C. 5 .

La leyenda también nos habla de las aventuras de Jasón y los Argonautas que cruzaron el estrecho con su navío Argos después de que Hércules diera muerte al rey troyano Laomedonte (padre de Príamo). Éstos luego tuvieron que atravesar la prueba de las Rocas Cianeas (azules) o Simplegadas (que se chocan), los dos escollos que guardaban la entrada al Ponto Euxino (Mar Negro).

El mito clásico fascinó al romántico Lord Byron. El 11 de abril de 1810, con sus jóvenes 22 años, George Gordon Byron (1788-1824) se embarcó en Esmirna en la fragata británica HMS Salsette procedente de la India y con destino final en Estambul. Al llegar a la boca del estrecho de los Dardanelos, y antes de visitar las ruinas de Troya, el 16 de abril, Byron y un teniente de la Salsette, de apellido Ekenhead ${ }^{6}$, tratando de rememorar el espíritu de Leandro, intentaron cruzar a nado el estrecho pero tuvieron que desistir a mitad de camino por la temperatura demasiado fría del agua.

4 Abidos fue una ciudad de la antigua Misia en el Helesponto, en el lugar de la punta Negara, en la parte asiática, en la orilla opuesta donde se encontraba Sestos. No confundir con la Abidos egipcia, una ciudad relacionada con la Dinastía XIX pero también con vestigios que se remontan al período Naqada I.

5 Museo (2003), Hero y Leandro. Introducción, texto, traducción y notas por Antonio Ruiz de Elvira. Madrid: Consejo Superior de Investigaciones Científicas.

6 William Ekenhead, nativo de Belfast (Irlanda), falleció ese mismo año de 1810 durante una desafortunada caída ocurrida en las fortificaciones de la isla de Malta. 
Un segundo intento tuvo lugar en la tarde del 3 de mayo ahora con el agua más cálida. El buque de guerra británico había anclado en Çanakkale para obtener el correspondiente permiso de las autoridades para dirigirse a la capital otomana. Aprovechando este intervalo, un esquife de la fragata llevó a los intrépidos nadadores a la orilla europea y desde allí se arrojaron a las cristalinas aguas. Esta vez la travesía fue exitosa y Ekenhead la realizó en una hora y cinco minutos, seguido de Byron cuya marca fue de una hora y diez minutos, toda una proeza teniendo en cuenta que el poeta sufría de deficiencias motrices reales, con serias deformaciones óseas en sus pies.

Pocos días después, el 9 de mayo, el poeta nacido en Londres compuso su "Written After Swimming from Sestos to Abydos" (Escrito después de nadar desde Sestos a Abidos), un poema para exaltar su zambullida y en homenaje a la historia de Hero y Leandro?.

\section{El cruce de los persas}

Es más conocido el cruce del estrecho por el ejército persa de Jerjes II en los comienzos de la Segunda Guerra Médica. El objetivo era invadir a las ciudades-estado de la Hélade. Las hordas persas avanzaron desde Sardes ${ }^{8}$ hasta el Helesponto. Fue entre Sestos y Maditos (la actual Eceabat) donde terminaban los dos puentes de barcas (a lo largo de unos dos mil metros) que Jerjes ordenó construir desde Abidos para cruzar el Helesponto con sus tropas en el 480 a. C. Heródoto nos narra con su pluma anecdótica las vicisitudes que enfrentaron las huestes asiáticas, procedentes de Sardes (la antigua capital de Lidia; hoy, Sar, en la provincia de Manisa, Turquía), que sumaban unos ciento cincuenta mil hombres (Robert Morkot: 1996, p. 76) entre soldados y portadores: «Cuando el doble puente había sido tendido, estalló una violenta tempestad que rompió los cables y dispersó los navios. Al tener noticias de ello, Jerjes montó en cólera y mandó que propinasen al Helesponto trescientos latigazos y que arrojaran

7 George Gordon Byron (1993), Selected Poems. Courier Corporation, p. 12.

8 Ciudad fundada por Giges (reinó entre 680-644 a. C.) como capital de Lidia. Jonios y ateniense la incendiaron en 499 a. C. Paso luego a ser la capital de la provincia romana de Asia a partir de 129 a. C. Reconstruida después de un terremoto en 17 d. C., fue desapareciendo lentamente luego de las depredaciones del rey sasánida Cosroes II (617). En 1407, Tamerlán la destruyó definitivamente. Heródoto pensaba que los etruscos procedían de la Sardes lidia. 
al agua un par de grilletes. [...] Jerjes, como digo, ordenó castigar al mar con esos correctivos, y, además, que les cortaran la cabeza a quienes habian dirigido la construcción de los puentes sobre el Helesponto»?.

Poco después, «y, al ver plagado de navíos todo el Helesponto, y atestados de soldados todas las playas y todos los campos de los abidenos ${ }^{10}$, en ese momento Jerjes se consideró un hombre afortunado; pero, acto seguido, se echó a llorar.» $\mathrm{Al}$ indagarlo su tío paterno Artábano sobre la razón de ese llanto, el "rey de reyes" confesó: "Es que me ha invadido un sentimiento de tristeza al pensar en lo breve que es la vida de todo ser humano, si tenemos en cuenta que, de toda esa cantidad de gente, no quedará absolutamente nadie dentro de cien años» ${ }^{11}$.

\section{La batalla de Egospótamos}

En el marco de la Guerra del Peloponeso (431-404 a. C.) entre Atenas y Esparta, se sucedieron una serie de combates navales entre espartanos y atenienses. En 411, frente al promontorio de Cinosema (Quersoneso tracio), la escuadra ateniense comandada por Trasíbulo y Trasilo derrotó a la peloponesia de Míndaro. Al año siguiente, en el 410, en Cícico, la flota ateniense ahora comandada por Alcibíades, Trasíbulo y Terámenes destruyó completamente a la flota espartana del almirante Míndaro que pereció en el combate.

Pero apenas cinco después, en el año 405, Atenas experimentó su peor derrota. En la desembocadura del Egospótamos, río del Quersoneso, al norte de Sestos, frente a Lámpsaco, en medio del estrecho, los espartanos (180 naves) liderados por el navarca Lisandro doblegaron a los atenienses (170 trirremes) guiados por el estratega Conón. Luego del combate, Lisandro se ensañó con

9 Heródoto (2006), Historia IV. Traducción y notas de Carlos Schrader. Madrid: Editorial Gredos; Libro VII, Capítulo 33, pp. 69-70.

10 El geógrafo Estrabón, haciéndose eco de la Ilíada, dice que el séptimo distrito dinástico de Troya abarcaba la región de quienes habitaban entre el río Esepo y la ciudad de Abidos, habitantes a quienes califica de troyanos porque todos los territorios en torno a Abidos estaban al mando de un personaje legendario, Asio el Hirtácida. La tradición la considera fundada por colonos de la isla de Lesbos, tal vez en el siglo VII a. C.

11 Heródoto:2006; Libro VII, Capítulo 45, p. 82. 
los vencidos y ordenó la muerte del estratega Filocles y de tres mil prisioneros atenienses ${ }^{12}$. Una vez más, los sonidos lúgubres de la guerra habían conmovido a los Dardanelos.

\section{La travesía de Alejandro}

Otro momento culminante de esta cronología sobre los Dardanelos es cuando Alejandro el Grande pisó la tierra de Asia, que ya nunca dejaría, hasta su muerte en Babilonia. Alejandro no iba a la guerra "a mirar" (como muchos reyes antiguos o generales modernos): era el adelantado en la ofensiva y el último en la retirada; si sus hombres pasaban frío, hambre o sed, él también lo padecía. Por eso, cuando en mayo del año 334 a. C. cruzó el Helesponto, fue el primero en llegar a la orilla asiática.

Ruth Sheppard, profesora del St. John's College de Oxford, nos cuenta cómo tuvo lugar ese episodio: «Después de llegar a Sestos Alejandro dejó en manos de Parmenión ${ }^{13}$ la supervisión del tránsito por el estrecho hasta Abidos en suelo asiático, a poco más de $1 \mathrm{~km}$ a través del Helesponto. Tras organizar la logística de esta operación a su segundo, Alejandro aprovechó la ocasión para ir a Troya en una visita cargada de trascendencia simbólica. En Eleo, en el extremo meridional del Quersoneso, ofreció un sacrificio en la tumba de Protesilao, de quien se decía había sido el primero de los soldados griegos que puso el pie en Asia durante la guerra de Troya. Atravesando el estrecho hacia el puerto aqueo, Alejandro timoneó él mismo la nave y sacrificó un toro a Poseidón y a las Nereidas, además de verter libaciones en el Helesponto para atraerse la buena fortuna. Diodoro dice incluso que, al desembarcar, Alejandro arrojó su lanza hacia Asia y saltó a tierra por delante de sus compañeros para dar a entender "que había recibido Asia de los dioses como un trofeo ganado por la lanza”. En la misma Troya se realizaron nuevos sacrificios y dedicatorias. Alejandro entregó su propia armadura, que cambió por alguna de las dejadas en el templo de Atenea Ilias en tiempos de la guerra troyana. Visitó las tumbas

12 Donald Kagan (2009), La Guerra del Peloponeso. Traducción de Alejandro Noguera. Barcelona: Edhasa; Parte VII: La caída final de Atenas, 405-404, p. 704 y ss.

13 Parmenión (400-330 a. C.), general macedonio al servicio de Filipo II y de su hijo Alejandro. Se destacó en las batallas de Gránico, Issos y Gaugamela durante la conquista del Imperio Aqueménida. 
de Aquiles y otros héroes homéricos con veneración y ofrendas, y apaciguó al espíritu del rey troyano Príamo con otro sacrificio» ${ }^{14}$.

\section{El infructuosos acoso árabe de Constantinopla}

En la primavera boreal del año 649 (28 de la Hégira) una flota musulmana partió del puerto de Akka (Palestina) al tiempo que otra se hacía a la mar desde las costas egipcias; ambas tenían como objetivo la isla de Chipre. El artífice de esta expedición marítima, considerada como la primera que los musulmanes realizaron en su historia naval y en el Mediterráneo, fue Mu'áwiyah Ibn Abi Sufyán (ca. 602-680), a la sazón gobernador de Siria y años más tarde primer califa omeya; él mismo la dirigió en persona. Los historiadores árabes, especialmente al-Baladhuri (muerto en 892), se hacen eco del empeño del fundador de la dinastía omeya en realizar esa primera empresa naval contra una isla que estaba bajo dominio bizantino y llama la atención sobre su insistencia en convencer primero al segundo califa Umar Bin al-Jattáb (gobernante entre 634-644) y después al tercer califa Uzmán Ibn Affán (g. 644656) para realizarla.

Esta primera incursión naval musulmana en el Mediterráneo oriental posibilitó que 25 años después, cuando Mu'áwiyah era el primer soberano del califato omeya, se desencadenara una serie de ataques contra la capital bizantina. Entre los años 674 y 678, los árabes lograron sitiar Constantinopla. Dirigidos por líderes como el propio hijo del califa, Yazid Ibn Mu'áwiya Ibn Abi Sufyán (sucesor de su padre a partir de 680), Gunada Ibn Abu Umayya, Fadhala Ibn Ubayd y el almirante Yazid Ibn Shagara, los shalandi (navíos de los musulmanes), luego de costear el litoral egeo de Anatolia, penetraron por los Dardanelos y establecieron una base de operaciones en la península de Cícico en Mármara, el mar que los griegos antiguos llamaban Propóntide (de pro, «antes/anterior»y Pontos, «mar»).

Luego de varios ataques navales y terrestres, donde en uno de ellos pereció Abu Ayyub al-Ansari (ca. 576-674), compañero del Profeta Muhammad, la flota musulmana recibió fuertes daños por un arma secreta y desconocida hasta entonces inventada por el ingeniero Calínico de Heliópolis (Baalbeck,

14 Ruth Sheppard (2011), Alejandro Magno: Guerras. Traducción de Antonio Rincón Córcoles. Madrid: Editorial Libsa, pp. 100-101. 
Líbano) en 672. El emperador Constantino IV (reinante entre 661-685) había equipado a sus dromones (naves de combate) con una especie de sifón que arrojaba una sustancia incendiaria de nuevo desarrollo: el fuego griego (un compuesto inflamable hecho con petróleo proveniente del Cáucaso) ${ }^{15}$. Al mismo tiempo el ejército musulmán de socorro comandado por Sufyán Ibn Awf era derrotado en el Asia Menor por el general bizantino Floro ${ }^{16}$.

Esta serie de reveses obligó a la flota árabe a abandonar el sitio y replegarse a sus bases en Siria, pero en el camino de vuelta fue casi aniquilada por una tormenta cerca de la costa de la antigua Panfilia (la actual Antalya, Turquía).

El segundo y último sitio árabe de Constantinopla tuvo lugar entre mediados de agosto de 717 y mediados de agosto de 718 . Fue llevado a cabo por Maslama Ibn Abd al-Malik (m. 738), hijo del califa omeya Abd al-Malik Ibn Marwán (r. 685-705), el constructor de la Cúpula de la Roca en Jerusalén. Maslama fue un estratega militar que tuvo en jaque constante al ejército bizantino entre 705-714 y cumplió funciones de gobernador militar en Armenia y Azerbaiyán (más tarde sería gobernador de Irak y del Cáucaso).

Esta nueva expedición contó con un ejército que cruzó el Helesponto y avanzó hacia la capital bizantina y una flota que penetró por los Dardanelos. Pero una vez más las formidables murallas de Constantinopla y el fuego griego arrojado por los navíos bizantinos frustraron el emprendimiento y Maslama fue forzado a retirarse con graves pérdidas ${ }^{17}$.

15 Véase Angus Konstam and Peter Dennis (2015), Byzantine Warship vs Arab Warship: 7th-11th Centuries. Oxford: Osprey Publishing.

16 Theophilus of Edessa (2011), Theophilus of Edessa's Chronicle and the Circulation of Historical Knowledge in Late Antiquity and Early Islam. Translated with an introduction and notes by Robert G. Hoyland. Liverpool: Liverpool University Press, pp. 166-167.

17 Véase Paul K. Davis (2001), “Constantinople: August 717-15 August 718”. 100 Decisive Battles: From Ancient Times to the Present. Oxford, United Kingdom: Oxford University Press. pp. 99-102; Rodolphe Guilland (1959), "L'Expedition de Maslama contre Constantinople (717-718)". Études byzantines. Paris: Publications de la Faculté des Lettres et Sciences Humaines de Paris, pp. 109-133. 
En el verano boreal de 904, dos griegos conversos al Islam, Rashiq al-Wardami (m. 921/2), llamado originalmente León de Trípoli, y Damyanah (m. 924), conocido como Damián de Tarso, con una flota musulmana de 54 bajeles pertenecientes a las capitanías de Siria y Egipto, intentaron un ataque contra Constantinopla. Luego de cruzar los Dardanelos, saquearon Abidos ante la impasible actitud de la escuadra bizantina del almirante Eustathios Argyros que rehuyó el combate. Cuando el emperador León VI el Sabio reemplazó a Argyros por Himerios, un comandante expeditivo y muy agresivo, al-Wardami y Damyanah optaron por volverse atrás y navegaron hacia el oeste hasta Salónica, la segunda gran metrópolis bizantina. Esta resultó ser una presa fácil.

Entre el 29 y el 31 de julio la urbe portuaria tesalonicense fue saqueada. Los musulmanes capturaron sesenta naves, se alzaron con un riquísimo botín, y con veintidós mil cautivos, entre ellos un testigo presencial, Ioánnis Kaminiates, quien más tarde redactaría la crónica "La captura de Salónica"18. La flota musulmana regresó a través del Egeo hasta la isla de Creta, sede de un emirato fundado por musulmanes cordobeses ${ }^{19}$, que le servía de base estratégica

18 Véase John Kaminiates (2000), “The capture of Thessaloniki” (D. Frendo, A. Fotiou, and G. Böhlig, trans.), Byzantina Australiensia, 12. Perth: Australian Association for Byzantine Studies.

19 Entre 814 y 818, unos quince mil hispanomusulmanes se vieron forzados a abandonar su Córdoba natal por oponerse al emir omeya al-Hákam I. Primero llegaron a Alejandría (Egipto). Pero allí tuvieron también problemas con las autoridades abbasíes que controlaban la región. Parece ser que hacia 824, el gobernador abbasí les proporcionó naves y pertrechos para que se hicieran a la mar y conquistaran Creta, que era una posición naval y militar bizantina que amenazaba desde hacía dos siglos los enclaves costeros musulmanes del Mediterráneo oriental. Los andalusíes cordobeses que llegaron a Creta eran en su gran mayoría musulmanes muladíes (hispanorromanos y godos conversos al Islam) y bereberes. Audaces y liderados por sus propios fuqahá (jurisconsultos) desembarcaron en la legendaria isla del rey Minos, vencieron la resistencia bizantina y fundaron en la costa norte la ciudadpuerto de Candía. Así establecieron el Emirato de Creta (827-961). El especialista griego Vassilios Christides en su disertación erudita señala que "siguiendo el patrón islámico, ningún tipo de islamización a la fuerza fue aplicado en la recién conquistada tierra de Creta". Las evidencias arqueológicas indican claramente que las iglesias permanecieron intactas y que la Basílica de Gortyna fue restaurada en el siglo IX durante la ocupación musulmana. En Candía, musulmanes y cristianos trabajarán codo a codo como artesanos y mercaderes. Véase Vassilios Christides (1984), The Conquest of Crete by the Arabs (ca. 824): A Turning Point in the Struggle between 
para operaciones en el Mediterráneo oriental. Precisamente, en 912, León VI el Sabio intentó conquistar Creta a los musulmanes, pero la invasión fracasó y la flota bizantina se vio obligada a retirarse. Finalmente en 961, el comandante bizantino Nicéforo Focas (912-969), elegido emperador en 963, conquistaría el emirato cretense.

\section{Los otomanos se posicionan en el estrecho}

Entre 1297 y 1360, el beylicato ${ }^{20}$ de Karasi tuvo el control de las actuales provincias turcas de Balikesir y Çanakkale con epicentro en Bergama (la antigua Pérgamo). Estos turcos oguz se convirtieron en una potencia naval en el mar Egeo y los Dardanelos. Karasi sería el primer beylicato (en turco, beylik) conquistado por Osman Gazi (r. 1299-1326), cuyo propio beylicato daría origen al Imperio Otomano. La adquisición de Karasi permitió a los otomanos comenzar la conquista de las tierras europeas en Rumelia ${ }^{21}$ a través de los Dardanelos.

El sultán otomano Orhán I (r. 1326-1359) será el que logre dominar el área vecina a los estrechos. Ya en 1346, «... Orhán cruzó los estrechos y llegó a la Tracia con un ejército de 5.500 soldados... $\rangle^{22}$.

Y en 1354, el hijo de Orhán, Solimán Pashâ, ocupó Gallipoli (evacuada por su población griega a raíz de un terremoto), facilitando al Estado otomano una cabeza de puente en Europa continental. A partir de entonces el paso vital

Byzantium and Islam. Athens: Academy of Athens; Vassilios Christides, "Relaciones entre Creta bizantina y los omeyas de Siria y al-Andalus", en VV.AA. (2001), El esplendor de los omeyas cordobeses: La civilización musulmana de Europa occidental. Vol. 1. Granada: Fundación El Legado Andalusí, pp. 62-67.

20 Los beylicatos anatólicos (en turco, Anadolu Beylikleri) fueron algo así como los Reinos de Taifas turcos en Anatolia. Esto es tan así que los otomanos turquizaron la expresión árabe Muluk at-Tawa'if y la transformaron en Tevâif-y muluk para denominar los territorios de estos régulos o beys anatólicos.

21 Rumeli es la variación turca de Imperio Romano, nombre que significa «tierra de romanos». La Rumelia era para los turcos la península de los Balcanes. En un sentido más restringido, identifica Macedonia y Tracia con la inclusión de la península de Gallipoli.

22 Francisco Veiga, El turco: Diez siglos a las puertas de Europa. Barcelona: Debate, 2011, p. 107. 
que conduce del Egeo al Mármara permanecerá hasta nuestros días en poder de los turcos.

Luego de tomar Constantinopla en 1453, una de las primeras medidas llevadas a cabo por el sultán Mehmet Fatih (r. 1451-1481), que sería el conquistador de la capital bizantina, fue asegurar los Dardanelos, único paso por donde podría venir una amenaza del Occidente latino.

Hacia 1461, el sultán otomano ordenó a Yaqub, gobernador de Gallipoli y el Quersoneso ${ }^{23}$, construir dos fortalezas en la parte "más estrecha del estrecho": Kilitbahir Kalesi (Castillo "cerrojo del mar), en la orilla europea (hoy Eceabat), un nombre que habla claramente sobre su propósito de defensa, y Kale-i Sultaniye (Castillo del sultán), también conocido como Çimenlik Kalesi, en la actual Çanakkale (en turco, 'Castillo de la taza', por la forma de la fortaleza), sobre la orilla asiática. A partir de entonces, los Dardanelos estuvieron guardados por dos baluartes considerados inexpugnables ${ }^{24}$.

\section{Un poeta inglés muerto en Skyros}

Hace 101 años, en enero de 1915, un absurdo plan ideado por Winston S. Churchill, Primer Lord del Almirantazgo, provocó que unos 570 mil soldados británicos, australianos, neozelandeses y franceses invadieran la península de Gallipoli defendida por los 315 mil hombres del quinto ejército otomano. Entre el 25 de abril de 1915 y el 9 de enero de 1916, o sea durante 8 meses, 2 semanas y 1 día, la guerra demostró, una vez más, ser la mayor desgracia de la humanidad. A pesar de disponer de la marina más poderosa del mundo y de la mejor tecnología militar de la época, la fuerza atacante se vio obligada a retirarse abrumada por las bajas sufridas en las estrechas y tortuosas playas y en el mar.

23 El término griego "jersónisos" quiere decir isla continental, es decir, "península". En el mundo griego antiguo hubo varias penínsulas que recibieron este nombre. Los más famosos Quersonesos fueron: el Tracio o Helespóntico; el Táurico o Traqueo, en el mar Negro; el Corintio; el Cario en donde fueron fundadas las ciudades de Halicarnaso y Cnido; y el Rodio también en Caria, al sur del anterior y frente a la isla de Rodas.

24 Citado por David Nicolle and Adam Hook (2010), Ottoman Fortifications 13001710. Oxford: Osprey Publishing, p. 17. 
Al menos 65.000 soldados aliados (británicos, franceses, australianos, neozelandeses, senegaleses e indios) murieron en la campaña y otros 140.000 quedaron heridos de los que muchos murieron en las semanas y meses siguientes. Un número similar de bajas tuvieron los turcos. Una historia extraordinaria y aterradora en un lugar cercano a las ruinas de Troya donde, según los poetas eduardianos, los antiguos héroes se despertaron en medio del tronar de los cañones. Así nació la frase "No se puede pasar Çanakkale" (en turco, Çanakkale geçilmez): es decir, es imposible por la fuerza cruzar los Dardanelos.

La serie de batallas navales y terrestres que se sucedieron en los Dardanelos en 1915 contienen numerosas anécdotas y vivencias de los hombres que fueron a morir allí, absurdamente, por "el rey y por la patria" (King and Country) y, paradójicamente, por el diletantismo de Churchill y la inconmensurable obstinación, incompetencia y vanidad de los generales y almirantes británicos. La idea y la planificación de la campaña de Gallipoli fueron concebidas con la misma mentalidad de aquellos que diseñaron las batallas de Ypres, Verdún y del Somme: «... los jefes elegidos para conducirla eran demasiado viejos, o carecían de experiencia, y a veces padecían ambas cosas a la vez. Fue su ignorancia, su mala dirección y su testarudez lo que provocó el fracaso de la expedición» ${ }^{25}$.

La loca idea de Churchill de asaltar los Dardanelos fue duramente cuestionada desde un principio por el entonces Primer Lord del Almirantazgo de la Marina Real Británica, el almirante John Arbuthnot Fisher (1841-1920). Éste, el 19 de enero de 1915 —en una confesión al almirante Sir John Jellicoe (1859-1935), el mismo que se haría famoso en la Batalla de Jutlandia del año siguiente-, había exclamado con una mezcla de ira y pesadumbre: "Abomino a los Dardanelos"26.

Una de esas tristes historias es la del subteniente Rupert Brooke (18871915), nacido en la localidad de Rugby, famosa por haberse inventado allí en 1823 ese viril deporte de conjunto. Alumno del King's College de Cambridge, se destacó por sus dotes de poeta, su fina elegancia y buen vivir. El poeta irlandés

25 John Macdonald (1989), Grandes Batallas del Mundo. Barcelona: Folio; "Bahía de Suvla/6 de agosto 1915-9 de enero 1916", p. 138.

26 Graham T. Clews (2010), Churchill's Dilemma: The Real Story Behind the Origins of the 1915 Dardanelles Campaign. Santa Barbara, California: Praeger, p. 123. 
William Butler Yeats (1865-1939) llegó a llamarlo “el joven más guapo de Inglaterra" ${ }^{27}$ Brooke embarcó en la Expedición Británica Mediterránea contra los Dardanelos el 28 de febrero de 1915. En algún camarote o sobre el puente escribirá estas sentidas estrofas que anunciaban la catástrofe:

They say Achilles in the darkness stirred...

And Priam and his fifty sons

Wake all amazed, and hear the guns, And shake for Troy again.

Dicen que Aquiles en la oscuridad se revolvió...

Y que Príamo y sus cincuenta hijos

Se despiertan asombrados, y escuchan los cañones.

$\mathrm{Y}$ vuelven a temblar por Troya ${ }^{28}$.

«La popularidad sin rival de Homero en la imaginación de finales de la era victoriana y de la era eduardiana quizá refleje el papel de la Ilíada en el sistema educativo público inglés. En el momento álgido del Imperio británico, Homero tal vez fuera el poeta que hablaba con mayor emoción a los imperialistas británicos, tanto por su "caballerosidad" y su "entereza" ante la muerte (sin olvidar su énfasis en el atletismo y la resistencia) como por su glorificación del coraje en la guerra. Tanto si era en la sábana surafricana, como en las trincheras de Flandes o los cielos de Picardía, Homero evocaba las más potentes imágenes en aquellos jóvenes que habian sido educados para considerarse a sí mismos los nuevos atenienses. Durante la Primera Guerra Mundial, el escritor inglés Maurice Baring ${ }^{29}$ escribió sobre:

\section{Combates tales que el ciego Homero nunca cantó, Que ni Héctor ni Aquiles jamás conocieron, En lo alto del cielo vacío.}

27 Michael Hastings (1967), The Handsomest Young Man in England: Rupert Brooke. London: Michael Joseph, p. 12.

28 Sir Edward Howard Marsh (1918), Rupert Brooke: A Memoir. London: John Lane Company, p. 177.

29 Maurice Baring (1874-1945), diplomático, lingüista, periodista, poeta, dramaturgo y novelista. Véase Maurice Baring (2012.), Poems: 1914-1919. London: Forgotten Books. 
Pero inevitablemente fue en Gallipoli donde Homero dio de pleno en el blanco, porque Troya y el cabo Helles están una frente al otro separado por el estrecho de los Dardanelos. Alli era imposible que los jóvenes poetas y escritores del Imperio británico, como John Masefield, A. P. Herbert, Patrick Shaw-Stewart Compton Mackenzie, no pensasen en la Ilíada. Al ver morir a sus amigos en las trincheras de Suvla, estando él mismo gravemente herido, el joven francés Jean Giraudoux ${ }^{30}$ extrajo también su inspiración del terrible trauma sufrido para el drama 'La guerra de Troya no tendrá lugar': “Por qué contra nosotros?", dice Héctor. "Troya es famosa por su arte, su justicia y su humanidad"»".

Rupert Brooke contrajo sepsis al ser infectado por la picadura de un mosquito. Murió a las 16:46 del 23 de abril de 1915 en un barco hospital francés amarrado en un muelle de la isla griega de Skyros $^{32}$. A las 23 horas de ese mismo día sería sepultado bajo un olivar de la isla por indicación de su compañero William Denis Browne (1888-1915), que moriría en acción dos meses después en Gallipoli.

El entonces teniente de navío Patrick Houston Shaw-Stewart (17 de agosto de 1888 - 30 de diciembre de 1917), también poeta y alumno de Eton y Oxford, cumplió un papel destacado en el funeral de Brooke. Y aunque logró sobrevivir para contarlo, finalmente cayó abatido en los campos de Francia dos años después. Será el autor de uno de los poemas más recordados de la Primera Guerra Mundial: "Aquiles en la trinchera", en inglés Achilles in the Trench ${ }^{33}$.

30 El escritor Hippolyte Jean Giraudoux (1882-1944) fue condecorado con la Legión de honor a título militar durante la Primera Guerra Mundial. Su drama La guerre de Troie n'aura pas lieu fue estrenado en el Théâtre de l'Athénée de París, el 21 de noviembre de 1935. En esa obra actuó Maria Falconetti (Andromaca), actriz francesa que fallecería en Buenos Aires el 12 de diciembre de 1946.

31 Michael Wood (2013), En busca de la guerra de Troya. Traducción castellana de Silvia Furió. Barcelona, Crítica, pp. 51-52.

32 Isla griega del mar Egeo, una de las Espóradas. El historiador y militar ateniense Tucídides menciona que la isla estaba habitada por la tribu tracia de los dólopes y fue conquistada por Cimón después de las guerras Médicas en 496 a. C. Luego pasó por muchas manos (romanos, bizantinos, venecianos y otomanos) hasta ser recuperada por Grecia en 1830.

33 Véase Elizabeth Vandiver (2010), Stand in the Trench, Achilles: Classical Receptions in British Poetry of the Great War. Oxford: Oxford University Press. 
Cuando en 1915 Shaw-Stewart estaba estacionado en la isla de Imbros $^{34}$ esperando ser enviado al infierno de Gallipoli, redactó estos versos mientras observaba a lo lejos la colina de Hisarlik sobre la costa anatólica, cuna de la legendaria Troya:

Ví a un hombre esta mañana

Que no deseaba morir:

Me pregunto y no puedo responder, Si yo deseo morir.

Hermoso rompió el día esta mañana

Contra los Dardanelos;

La brisa sopló suave, las mejillas

Estaban frías como conchas de mar.

Pero otras conchas me esperan a través del mar Egeo,

Metralla y explosivos,

Proyectiles e infiernos.

Oh infierno de buques y ciudades, Infierno de hombres como yo.

Fatídica segunda Helena,

¿Por qué debo seguirte?

Aquiles llegó a Troya

Y yo al Quersoneso:

Él pasó de la ira al combate,

Y yo de tres días de paz.

¿Fue tan difícil, Aquiles?

¿Tan difícil es morir?

Tú supiste y yo no sé:

Soy, pues, el más feliz.

34 Llamada en turco Gökçeada, es una isla del mar Egeo que hoy pertenece a Turquía. Es la mayor isla del país y forma un distrito de la provincia de Çanakkale. Mencionada por Homero en la Ilíada, fue incorporada al Imperio persa junto con Lemnos por el general Ótanes en el siglo VI a. C. Según Heródoto, en aquel momento ambas islas estaban habitadas por los pelasgos. 


\section{Regresaré esta mañana \\ Desde Imbros por el mar; Quédate en la trinchera, Aquiles, Envuelto en llamas, y lucha por mín.}

En nuestros días nadar de Europa al Asia, y viceversa, se ha convertido en un deporte cotidiano para turistas audaces. Los émulos de Leandro y Lord Byron abundan y compiten todos los años en lograr la heroica proeza.

En 1926, el aventurero, viajero y gran nadador estadounidense Richard Halliburton (1900-1939) cruzó a nado el "Mar de Hele" emulando a su héroe, el poeta inglés.

En 1984, el historiador, escritor y militar inglés Patrick "Paddy" Leigh Fermor (1915-2011), amante de lo griego y autor de libros como "Mani: Viajes por el sur del Peloponeso" (1958) y "Roumeli” (1966), y que logró secuestrar al general Heinrich Kreipe, comandante alemán de la isla de Creta en 1944, cruzó a nado el Helesponto a los 69 años.

Y otra gran hazaña. El 30 de agosto de 2012, la estudiante georgiana Ani Lominadze, de apenas 17 años de edad, logró superar la distancia de cinco kilómetros y medio en 50 minutos entre las ciudades de Eceabat y Çanakkale nadando a través de los Dardanelos con las manos y los pies atados. El original estilo se llama "kolhuri" y se lo practicaba en la antigua Cólquida, la mítica tierra de Medea donde se encontraba oculto el vellocino de oro.

35 Michael Wood: 2013, pp. 52-53. 


\section{REFERENCIAS BIBLIOGRÁFICAS}

CURRAN, TOM (2015). The Grand Deception: Churchill and the Dardanelles. Newport, New South Wales (Australia): Big Sky Publishing.

DAVISON, ANDREW (2014). Border Thinking on the Edges of the West: Crossing Over the Hellespont. London and New York: Routledge.

FREELY, JOHN (2010). Children of Achilles: The Greeks in Asia Minor since the Days of Troy. London: I. B. Tauris.

HAMMOND, NICHOLAS G. L. (1996). “The construction of Xerxes' bridge over the Hellespont", The Journal of Hellenic Studies, [1996] 88-107.

KORFMANN, MANFRED (1996). "The Citadel and the Lower City of Troia at the Dardanelles. City of War and Peace Where Seas and Continents Meet," in Yildiz Sey (ed.), Housing and Settlement in Anatolia: A Historical Perspective (Istanbul 1996) 83-98.

STONEMAN, RICHARD (2011). Across the Hellespont: A Literary Guide to Turkey. London: I. B. Tauris.

VAN DER VAT, DAN (2010). The Dardanelles Disaster: Winston Churchill's Greatest Failure. New York: The Overlook Press. 